\title{
SECOND HARMONIC BEAM ANALYSIS, A SENSITIVE TECHNIQUE TO DETERMINE THE DURATION OF SINGLE ULTRASHORT LASER PULSES
}

\author{
C. KOLMEDER, W. ZINTH and W. KAISER \\ Physik Department der Technischen Universität München, München, Germany
}

Received 18 June 1979

\begin{abstract}
The second harmonic beam generated in a noncollinear arrangement allows the observation of the autocorrelation functio: A compact optical beam splitter and imaging system eliminates alignment problems. Single pulses of 1 ps duration and approximately $10^{-8}$ joule energy are readily observed using an optical multichannel analyser.
\end{abstract}

During the past decade the application of light pulses in the picosecond time domain has received increasing attention. In these investigations it is of primary interest to know the duration of the ultrashort light pulses. Two methods to measure the pulse duration have found frequent application: The two-photon fluorescence technique, TPF [1] and the streak camera [2]. The autocorrelation pattern of the TPF technique gives direct information on the pulse duration and the coherence properties of the pulse [3] but the difficult alignment, the high-power requirement, and the weakly emitting fluorescent pattern become a serious hindrance for wide application. The commercially available streak cameras are very expensive, show a jitter of the order of $100 \mathrm{ps}$ of more, and have a temporal resolution of several picoseconds (except very near to the special cut-off frequency).

In 1966 it was suggested [4,5] and experimentally demonstrated [4] that the second harmonic signal generated by two equal pulses in a nonlinear crystal allows the determination of the autocorrelation function giving a value for the duration of the ultrashort light pulse. The technique has found recent application for the studies of the pulse duration of passively mode-locked $\mathrm{CW}$ dye lasers or synchronously pumped $\mathrm{CW}$ dye lasers. The autocorrelation function is obtained by introducing quasi-continuously a delay between the two interacting pulses [6].

More recently, it was noticed that the spatial width of the second harmonic beam (SHB) may be used to obtain the autocorrelation function $[7,8]$, i.e., the du-

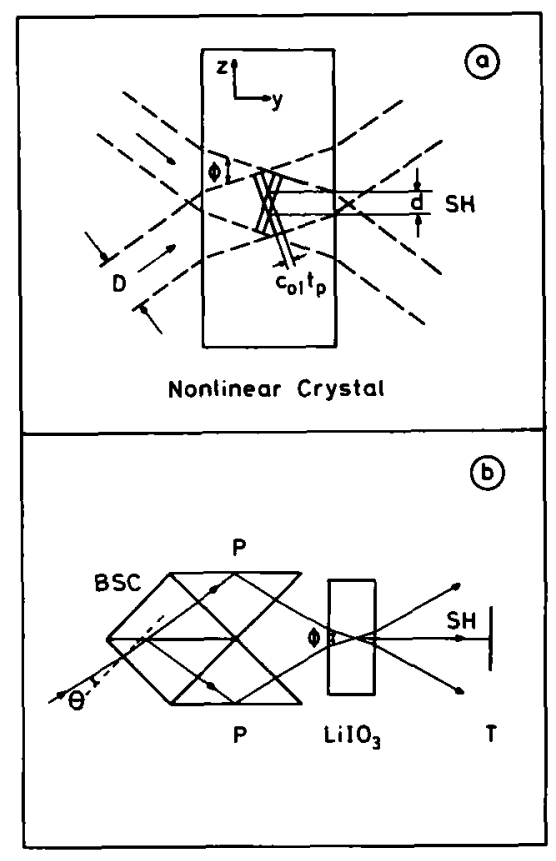

Fig. 1.a Schematic of the second harmonic beam technique. The two incident pulses with beam diameters $D$ cross in the nonlinear crystal at angle $\phi$. The spatial width $d$ of the second harmonic light beam provides directly the duration of the pulses $t_{\mathrm{p}} \cdot \mathrm{b}$ Schematic of a practical imaging device consisting of a beam splitting cube BSC and two attached prisms $P$. The distribution of the second harmonic beam is measured at the position $T$ by an optical multichannel analyser. The crossing angle $\phi$ can be changed by varying the entrance angle $\theta$. 
ration of a single laser pulse may be deduced from the intensity distribution of the second harmonic beam. The principle is shown in fig. 1a. Two pulses generated by a preceding beam splitter (wave vectors $\boldsymbol{k}_{\mathrm{L} 1}$ and $\boldsymbol{k}_{\mathrm{L} 2}$ ) cross in a nonlinear crystal at an angle $\phi$ and produce a second harmonic with wave vector $k_{2}$. The crystal has to be oriented in such a way that the phasematching condition $k_{\mathrm{L} 1}+k_{\mathrm{L} 2}=k_{2}$ holds. In general, the input pulses have ordinary and the second harmonic pulse has extra-ordinary polarisation. Simple geometry shows that the length of the pulses (the time information) is projected in the $z$-direction. For sufficiently large diameters, $D$, of the incoming beams the intensity distribution of the second harmonic beam in the $z$-direc: tion gives directly the autocorrelation function of the primary pulses. The spatial width, $d,(\mathrm{fwhm})$ and the pulse duration, $t_{\mathrm{p}}$, are related as follows:

$t_{\mathrm{p}}=\frac{\gamma d \sin (\phi / 2)}{c_{\mathrm{o} 1}}$

where $c_{01}$ is the group velocity of the incident light pulses with ordinary polarisation and $\gamma$ is a form factor depending upon the shape of the pulse. For gaussian pulses one has $\gamma=2^{1 / 2}=1.44$. It is important for the SHB method discussed here that the geometrical length of the light pulse, $L_{\mathrm{p}}=c t_{\mathrm{p}}$, is smaller than the beam diameter, $D$. For $L_{\mathrm{p}} \leqslant D / 3$ eq. (1) holds with good accuracy, for $L_{\mathrm{p}} \approx D$ a more complex equation involving the temporal and spatial shape of the light pulse has to be used [7].

This note is divided into four parts. (i) Various features of the technique such as temporal resolution and frequency dependence are examined. (ii) The direct observation of the second harmonic beam with an optical multichannel analyser is discussed and the required pulse energies are given. (iii) A rugged device for splitting the input pulse and directing the pulses into the nonlinear crystal is introduced. (iv) Experimental results are presented demonstrating the ease and precision of electronic data acquisition.

(i) It is readily seen from fig. 1a and from eq. (1) that the width of the second harmonic beam, $d$, increases for smaller crossing angles $\phi$. This fact allows us to adjust the time resolution to the experimental situation. The following example should illustrate the point. $\mathrm{LiIO}_{3}$ is assumed to be the nonlinear crystal and $\lambda=1.06 \mu \mathrm{m}$ the wavelength of the light pulse to be in- vestigated. A (gaussian) pulse with $t_{\mathrm{p}}=5 \mathrm{ps}$ gives a width of the second harmonic beam of $d=1.7 \mathrm{~mm}$ for $\phi=39.4^{\circ}$. The same width of the beam $d=1.7 \mathrm{~mm}$ is obtained for $t_{\mathrm{p}}=1 \mathrm{ps}$ when a crossing angle of $\phi=$ $7.7^{\circ}$ is used. Small angles $\phi$ give favorable time resolution for short pulses. (It should be recalled that changing $\phi$ leads to a new phasematching situation, i.e., the angle between the optical axis and the axis of the second harmonic beam has to be adjusted).

There are several factors which may affect the time resolution. The divergence of the input beams leads to a divergence of the second harmonic radiation. In addition, very narrow second harmonic beams may introduce a noticeable diffraction angle. To reduce these two angular contributions the distance between nonlinear crystal and detector target should be kept sufficiently small. In uniaxial crystals there exists a walkoff angle $\alpha$ between the $k$-vector of the extraordinary beam and the Pointing vector. Only for the case of $90^{\circ}$ phase matching the walk-off angle is zero. In $\mathrm{LilO}_{3}$ and a phasematching angle of $\theta \approx 40^{\circ}$ one calculates a maximum value of $\alpha \approx 5^{\circ}$. This angle leads for an interaction length $l$ to a broadening of the second harmonic beam of $\Delta z=l \times \sin \alpha$. In our experiments (see below) we work with $\mathrm{LiIO}_{3}, \phi=36^{\circ}, \theta=68^{\circ}, \alpha=2.5^{\circ}$, and $l=0.3 \mathrm{~cm}$. The beam broadening of $\Delta z \simeq 10^{-2} \mathrm{~cm}$ corresponds to a reduced accuracy in pulse duration of 0.1 ps.

Of special interest is the frequency range where the presented technique may be used. Certainly, the nonlinear crystal has to be transparent for the input and second harmonic beam and the color dispersion has to allow phasematching for practical angles. In table 1 several relevant parameters are listed. The crossing angles $\phi$ are calculated for $90^{\circ}$ phasematching (the entrance crystal surface is cut parallel to the optical axis). It is apparent from table 1 that $\mathrm{LilO}_{3}$ is well suited for the red and infrared part of the spectrum while $\mathrm{KDP}$ is more useful for shorter wavelengths. Potassium pentaborate, $\mathrm{KPB}$, a biaxial crystal, should allow the investigation of pulses at a wavelength as short as $435 \mathrm{~nm}$ [10] .

(ii) As indicated in fig. 1a, the generated second harmonic beam is well collimated. Its spatial distribution is readily observed without imaging device. For immediate observation of a single pulse it is convenient to use a vidicon system or, for more quantitative investigations, an optical multichannel analyser. The required pulse energy is of special importance for practical ap- 
Table 1

Relevant parameters for nonlinear crystals used for the SHB method a)

\begin{tabular}{|c|c|c|c|c|c|}
\hline Crystal & $\begin{array}{l}\text { Useful wavelength } \\
\text { range } \\
(\mu \mathrm{m})\end{array}$ & $\begin{array}{l}\text { Incident } \\
\text { wavelength } \\
(\mu \mathrm{m})\end{array}$ & $\begin{array}{l}\text { Nonlinear } \\
\text { coefficient } \\
\left(\mathrm{cm} \mathrm{dyn} \mathrm{d}^{1 / 2} \text { ) }\right.\end{array}$ & $\begin{array}{l}\text { Crossing angle } \phi \\
\text { for } 90^{\circ} \\
\text { phasematching }\end{array}$ & $\begin{array}{l}\text { Time } \\
\text { resolution b) } \\
\text { (ps) }\end{array}$ \\
\hline $\mathrm{LiIO}_{3}$ & $0.6-5.5$ & $\begin{array}{l}1.06 \\
0.6\end{array}$ & $d_{15}=1.32 \times 10^{-8}$ & $\begin{array}{l}39.4^{\circ} \\
16.1^{\circ}\end{array}$ & $\begin{array}{l}0.2 \\
0.1\end{array}$ \\
\hline ADP & $0.52-1.2$ & $\begin{array}{l}1.06 \\
0.53\end{array}$ & $d_{36}=1.26 \times 10^{-9}$ & $\begin{array}{r}21.0^{\circ} \\
4.4^{\circ}\end{array}$ & $\begin{array}{l}0.1 \\
0.02\end{array}$ \\
\hline KDP & $0.52-1.5$ & $\begin{array}{l}1.06 \\
0.53\end{array}$ & $d_{36}=1.04 \times 10^{-9}$ & $\begin{array}{r}20.3^{\circ} \\
6.1^{\circ}\end{array}$ & $\begin{array}{l}0.1 \\
0.03\end{array}$ \\
\hline
\end{tabular}

a) Data are taken from ref. [9]

b) Calculated for a spatial resolution of $0.07 \mathrm{~mm}$.

plications. In fig. 2, the energy value per pulse is plotted versus pulse duration for $\mathrm{LilO}_{3}$ and $\mathrm{ADP}$ crystals. The measurement of fig. 3 , where a pulse of $t_{\mathrm{p}}=1$ ps was investigated, was made with an input energy of $5 \times 10^{-9}$ joule using a $\mathrm{LiIO}_{3}$ crystal. The higher energy require-

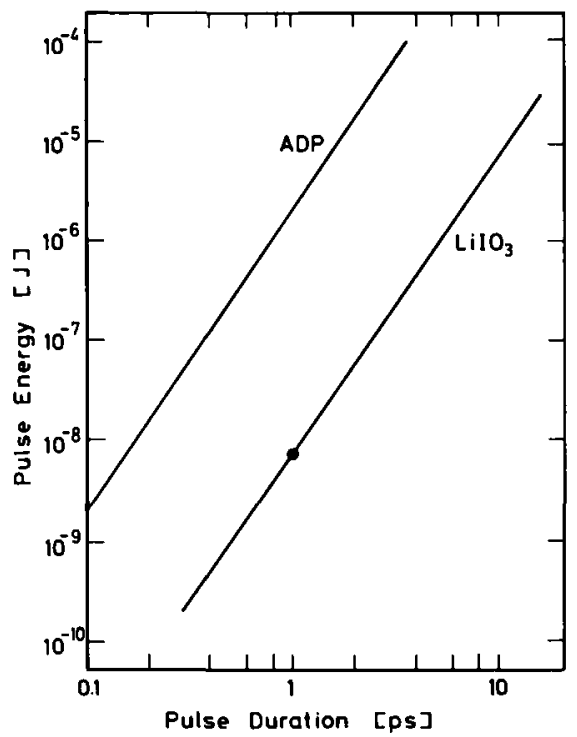

Fig. 2. Energy of the input pulse versus pulse duration obtained for a sensitivity of the optical multichannel analyser of 40 photons per count and channel. The lines are calculated for a wavelength of $1.06 \mu \mathrm{m}$ and for $90^{\circ}$ phasematching in $\mathrm{LiIO}_{3}$ and ADP crystals. The corresponding crossing angles are $39.4^{\circ}$ and $22.8^{\circ}$, respectively. The point marks an experimental result. ment of ADP follows from the smaller nonlinear coefficient of this material.

The pulse energy observed in our system is estimated as follows: A detector with a sensitivity (at $\sim 500 \mathrm{~nm}$ )

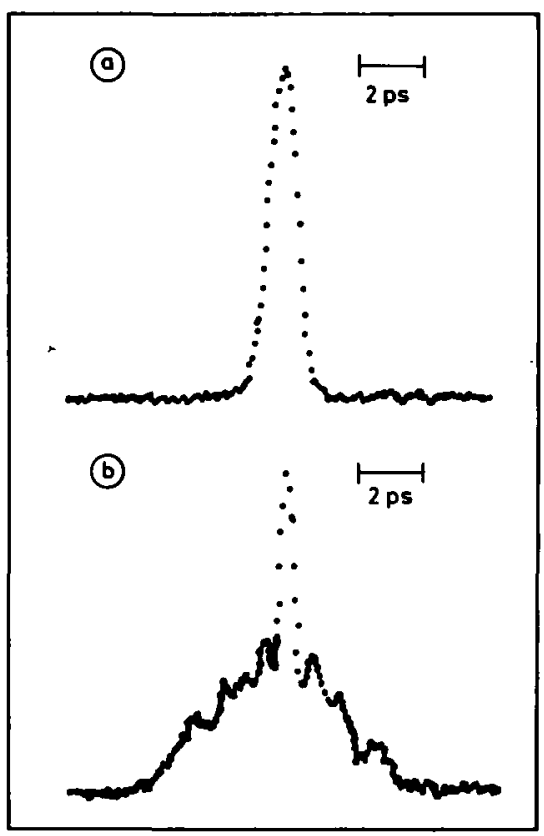

Fig. 3. Experimental pictures taken with an optical multichannel analyser. Fig. 3a shows the smooth autocorrelation function of a pulse selected from the rising part of the pulse train. The pulse duration is $1.0 \pm 0.2$ ps. Fig. $3 \mathrm{~b}$ represents a complex autocorrelation curve; the pulse is taken from the end of the pulse train. 
of 40 photons per count and channel gives good pictures with spatial resolution of $75 \mu \mathrm{m}$ when approximately 100 counts are received by 50 channels. Considering the losses of the total system to amount to a factor of ten (reflection losses of various surfaces and slit in front of tube) and assuming a conversion efficiency for the second harmonic generation of $10^{-4}$ one estimates $1 / 2 \times 10^{11}$ photons or $10^{-8}$ joule necessary for the input pulse. It should be noted here that tubes for multichannel analysers more sensitive by a factor of 10 are available making the observation of even weaker pulses possible.

(iii) As pointed out in the introduction the SHB technique requires the original pulse to be divided into two equal pulses. The two beams have to cross in the nonlinear crystal. Fig. 1b shows an optical system which performs this function with high precision. A beam splitter cube, BSC, generates two pulses with zero time delay and of approximately equal intensity; the two totally reflecting prisms, $P$, direct the two pulses into the nonlinear crystal. Different crossing angles $\phi$ are obtained by changing the incident angle $\theta$. Values of $\phi$ between $25^{\circ}$ and $45^{\circ}$ are readily adjusted with two prisms $P$ of apical angles of $90^{\circ}$. For smaller values of $\phi$, i.e. $0 \leqslant \phi \leqslant 30^{\circ}$ prisms with $60^{\circ}$ should be used. We have considered possible effects of the glass dispersion on the time resolution. Calculations show that for BK7 glass and for several centimeters of optical path the dispersion effects may be neglected even for pulses with $t_{\mathrm{p}} \approx 0.1 \mathrm{ps}$ at $\lambda=1.06 \mu \mathrm{m}$. We have used successfully the compact beam splitting device in a number of experiments. The very stable time delay, the ready change of the crossing angle $\phi$, and the ease of optical alignment make the system convenient as an accessory for routine pulse analysis.

(iv) Now we turn to the observation of the pulse duration of a single mode-locked laser pulse. The experimental system consists of an improved passively mode-locked Nd-glass laser operating at $\lambda=1.053 \mu \mathrm{m}$ [11]. Single light pulses were selected from different positions within the pulse train with the help of an electro-optic switch. The output beam of the laser oscillator was expanded by a factor of two to make the ratio beam diameter to pulse length, $D / L_{\mathrm{p}} \geqslant 3$. The laser pulse passed through the beam splitting device (discussed above) which generated a crossing angle of $36^{\circ}$ inside a $\mathrm{LilO}_{3}$ crystal. We used a $\mathrm{LiIO}_{3}$ specimen of $3 \mathrm{~mm}$ thickness and worked with a phasematching angle of $68^{\circ}$. Under these conditions the effect of beam walk-off is less than $0.1 \mathrm{ps.}$

In fig. 3 we present two pictures taken from the optical multichannel analyser. The laser pulses of figs. $3 a$ and $3 b$ were selected respectively from the rising part and from the end of the pulse train. It is well-known that bandwidth limited pulses occur only at the beginning of the pulse train $[3,12]$. In fact, we find a smooth autocorrelation function without background in fig. 3a. From the width of the second harmonic beam, i.e., from the number of channels at half maximum, we calculate a pulse duration of $1.0 \pm 0.2 \mathrm{ps}$. We point to the favorable signal to noise ratio which was obtained for an input energy of approximately $10^{-8}$ joule.

Fig. $3 \mathrm{~b}$ shows the pattern of a complex pulse originating from the decaying part of the pulse train. A sharp peak of 0.5 ps is superimposed on a broad background. It has been discussed by several authors that the many pathes of the intense pulse inside the dispersive laser resonator lead to severe break-up and partial loss of coherence of the light pulse. Figs. $3 a$ and $3 b$ clearly demonstrate the importance to work with the early pulses of a Nd-glass system and to avoid the application of the whole pulse train.

According to our experience, the SHB method provides approximately three orders of ten more photons to the optical detector than the TPF technique. The second harmonic generation in $\mathrm{LilO}_{3}$ is more effective than the two-quantum fluorescence in a diluted dye solution and the SHB is emitted highly directional while the TPF radiates into a solid angle of $4 \pi$. In other words, the large overall efficiency and the ease of optical alignment make opto-electronic detection and electronic data processing straight-forward.

\section{References}

[1] J.A. Giordmaine, P.M. Rentzepis, S.L. Shapiro and K.W. Wecht, Appl. Phys. Lett. 11 (1967) 216.

[2] D.J. Bradley, in: Ultrashort light pulses, ed. S.L. Shapiro (Springer Verlag, Berlin, Heidelberg, New York, 1977) p. 28.

[3] D. von der Linde, IEEE J. Quant. Electr. QE-8 (1972) 328.

[4] M. Maier, W. Kaiser and J.A. Giordmaine, Phys. Rev. Lett. 17 (1966) 1275.

[5] H.P. Weber, J. Appl. Phys. 38 (1967) 2231.

[6] E.P. Ippen and C.V. Shank, Appl. Phys. Lett. 27 (1975) 488. 
[7] J. Jansky, G. Gorradi and R.N. Gyuzalian, Optics Comm. 23 (1977) 293.

[8] R.N. Gyuzalian, S.B. Sogomonian and Z. Gy. Horvath, Optics Comm. 29 (1979) 239.

[9] S.K. Kurtz, in: Laser Handbook, eds. F.T. Arrecchi and E.O. Schulz-Dubois (North-Holland, Amsterdam, 1972);
B.F. Levine and C.G. Bethea, Appl. Phys. Lett. 20 (1972) 272.

[10] H.J. Dewey, IEEE J. Quant. Electr. Qe-10 (1976) 303.

[11] W. Zinth and C. Kolmeder, to be published.

[12] W. Zinth, A. Laubereau and W. Kaiser, Optics Comm. 22 (1977) 161. 